\title{
The characteristics of serious suicide attempters in Japanese adolescents- comparison study between adolescents and adults
}

\author{
Yoshitaka Kawashima, Takao Ito, Ryuichiro Narishige, Takuya Saito and Yoshiro Okubo*
}

\begin{abstract}
Background: Suicide is the leading cause of death among Japanese adolescents, and they may commit suicide differently from adults. However, there are few studies in medical-based data concerning adolescent patients seriously attempting suicide. We aimed to explore the characteristics of serious suicide attempts in Japanese adolescents, comparing them with those in adults.

Methods: We investigated adolescents who seriously attempted suicide and were treated at the Critical Care Medical Center (CCMC) of Nippon Medical School Hospital between 2000 and 2010, and we compared them with adult suicide attempters treated during 2009. We retrospectively studied medical records and collected clinical data and sociodemographic factors, including age, sex, psychiatric symptoms or diagnosis, methods of suicide attempt, motives for suicide attempt, previous deliberate self-harm, previous psychiatric history, parent loss experience, and previous psychiatric history in the family.

Results: Adolescent attempters were 15 males and 44 females, 13 to 18 years old (mean 16.39). Adult attempters were 37 males and 65 females, 19 to 79 years old (mean 39.45). In comparison to adult attempters, adolescent attempters were more frequently diagnosed with Borderline Personality Disorder (BPD), had more school problems and parent loss experience, but they had less financial problems. Gender differences between adolescents and adults were examined, and male adolescent attempters were found to be more frequently diagnosed with schizophrenia and had less financial problems than their adult counterparts, while female adolescent attempters were more frequently diagnosed with BPD, had more school problems and parent loss, but they had less previous psychiatric history than their adult counterparts.

Conclusions: Our findings indicated that adolescent attempters were more frequently diagnosed with BPD and had more school problems and parent loss experience but had less financial problems. Additionally, in male adolescent attempters, identifying patients with schizophrenia seemed important, as it was their most frequent psychiatric diagnosis. For female adolescents, adequately assessing family function and interpersonal conflicts seemed important, as they were more often diagnosed with BPD and had more school and family problems.
\end{abstract}

Keywords: Suicide attempts, Adolescents, Age differences, Critical care medicine

\section{Background}

The number of suicides in Japan has continued to exceed 30,000 every year, with the rate remaining at around 25.0 per 100,000 individuals since 1998, the highest rate among developed countries $[1,2]$. In adolescents aged $15-19$, the suicide rate is 2.4 per 100,000 individuals [3], with suicide being the leading cause of death [4]. In Japan, individuals 15-24 years old are the

\footnotetext{
* Correspondence: okubo-y@nms.ac.jp

Department of Neuropsychiatry, Nippon Medical School, 1-1-5, Sendagi, Bunkyo-ku, Tokyo 113-8602, Japan
}

most vulnerable group for suicide attempts and suicide mortality [5]. Suicide attempts in adolescents have been recognized as a major public health problem in not only Japan but also in other countries all over the world, because of their frequency, likelihood for recurrence, health care costs, and high risk for completed suicide [6]. In Japan, suicide behavior and suicide ideation among adolescents have been investigated in general community populations $15-24$ years old [7-9], but there are few studies of suicide attempters among adolescents to be found in medical-based data.

\section{() Biomed Central}


Suicide risk has been reported to be associated with psychiatric disorders [10-12], adverse childhood experiences [13], and family history [14]. Suicide attempts and especially repetition of attempts are high-risk factors for subsequent suicide [15-17]. Previous literature has indicated that $10-50 \%$ of adolescent suicide attempters reattempted [18], and that about $11 \%$ of these committed suicide [19]. Additionally, among individuals under 25 years of age, both those who committed suicide and those making serious suicide attempts were in a similar group with the same risk factors [20]. Therefore, studies of serious suicide attempters are as important as psychological autopsy studies investigating complete suicide attempters in consideration of suicide prevention. In addition, a survey of suicide attempters based on direct interviews makes it possible for us to learn more about their detailed characteristics, such as socio-demographic factors and psychiatric symptoms before their attempts.

Being in a developmentally transitional stage, adolescents will differ from adults. For example, during adolescence, they struggle to achieve body mastery, control sexual and aggressive urges, gain independence from the family, find new and appealing sexual relationships, and achieve a sense of identity [21]. Adolescents start to rely less on parents for support and more on their peers as they grow older [22]. Also, adolescents differ from adults in terms of their typical financial status, medical conditions, occupational responsibilities, coping styles, social support networks, and the stressors to which they are commonly exposed [18]. Furthermore, suicidal behavior among adolescents occurs in different contexts from older individuals [23]. For example, adolescent suicidal behavior often occurs in the context of family conflict, including strivings for autonomy, in the context of academic and disciplinary difficulties, and in the consequence of disruptions in peer relationships that are important as youths get older. These have been described in previous literature, which pointed out the importance of the human lifespan in consideration of measures to combat suicide as well as interventions for mental health problems of adolescents, including suicidality, that are not developmentally tailored [24].

Therefore, in the present study, we explored the characteristics of serious suicide attempters among adolescents admitted to a critical care medical center in Japan, comparing them with adults.

\section{Methods}

\section{Procedure}

This study was carried out at the Critical Care Medical Center (CCMC) of Nippon Medical School Hospital, Tokyo. Since all patients at CCMC are in medically serious and potentially fatal condition, they require a high level emergency care. Approximately 2000 patients per year are admitted to CCMC, and about 100 of them are suicide attempters (excluding complete suicides) per year. Consultation-liaison psychiatrists have been assigned to provide psychiatric services to all suicide attempters admitted to CCMC [25]. Psychiatrists evaluate patients on admission and periodically during their CCMC stay to manage their psychiatric problems [26].

In this study, we defined adolescents as 18 years old or younger, and adults as 19 years old or older. Adolescent suicide attempters admitted to CCMC totaled 59 during the 11-year period between January 2000 and December 2010. In this study, we regarded adult suicide attempters as the control group for comparison with adolescents. Additionally, the period of the adults was fixed at one year, as the adolescent sample size was small. We selected the 1-year period of January-December 2009, in which the gender ratio between adolescents and adults was most similar within the past five years (between 2006 and 2010). Thus we included 102 adult suicide attempters from 2009 in our study.

We conducted a retrospective study of medical records containing information about primary care or any prior psychiatric treatment. We collected the clinical data and socio-demographic factors of all participants, including age, sex, psychiatric symptoms or diagnosis, methods of suicide attempts, motives for suicide attempts, previous deliberate self-harm, previous psychiatric history, parent loss experience by age 18 , and previous psychiatric history in the family within a third degree of kinship.

In addition, we distinguished suicide attempts from non-suicidal self-injury without intent to die. We used definitions of suicide attempt based on the recent consensus on nomenclature for suicidology [27,28]. We defined suicide attempt as self-injurious behavior potentially resulting in fatality with at least some intent to end one's own life. Suicide attempt also was determined on the basis of previous desire to die and a history of suicide attempts.

\section{Assessment of psychiatric disorders}

Psychiatric diagnosis from medical records and information about any prior psychiatric treatment between January 2000 and December 2010 was based on DSM-IV and DSM-IV-TR by two or more experienced psychiatrists. In this study, psychiatric diagnosis with DSM-IV was remade according to DSM-IV-TR criteria. In case of disagreement with each other's psychiatric diagnosis, a decision was reached by mutual consent after holding discussions.

\section{Statistical analyses}

At the initial analysis, we examined the differences between adolescents and adults, using Pearson's chi- 
square test and Fisher's exact test for dichotomous and nominal variables. At the second analysis, males and females were analyzed separately because of the consistently significant gender differences in previous studies of adolescent suicide attempts [29]. We used a significance level of $p<0.05$ and two-sided probability. The SPSS version 16.0.2 statistical package (SPSS Inc., Chicago, IL., 2008) was used for the entire analysis.

\section{Ethics}

This study was approved by the ethics committee of Nippon Medical School Hospital, and conforms to the provisions of the Declaration of Helsinki.

\section{Results}

The demographic characteristics of all participants are presented in Table 1 . The adolescents consisted of 15 males (25\%) and 44 females (75\%), aged 13 to 18 , mean 16.39 years, and standard deviation $(S D) 1.5$ years. The number of adolescent suicide attempters increased with age. The adults consisted of 37 males (36\%) and 65 females (64\%), aged 19 to 79, mean 39.45 years, and standard deviation $(S D) 13.2$ years. As shown in Table 1, the number of female attempters was larger than that of the male attempters from adolescents to adults except for individuals 50-59 years old. There were no significant differences between the gender ratios of adolescents and adults

First we examined the differences between adolescents and adults (Table 2). Concerning psychiatric disorders, Borderline Personality Disorder (BPD) was seen more often among adolescents $\left(\chi^{2}(1)=4.42 ; p<0.05\right)$. Regarding motives for suicide attempts, school problems were more frequent among adolescents $\left(\chi^{2}(1)=20.87 ; p<0.001\right)$ and financial problems were more frequent among adults $\left(\chi^{2}(1)=8.33\right.$; $p<0.01)$. As for socio-demographic characteristics, adolescents had more parent loss experience $\left(\chi^{2}(1)=10.26\right.$; $p<0.05)$.

Then we examined the differences between adolescents and adults by gender (Table 2). For males, schizophrenia among psychiatric disorders was more often observed in adolescents (Fisher's exact test; $p<0.05$ ) and financial problems among motives were more often seen in adults (Fisher's exact test; $p<0.05)$. For females, $\operatorname{BPD}\left(\chi^{2}(1)=6.36\right.$; $p<0.05$ ), indeterminate (Fisher's exact test; $p<0.05)$ among psychiatric disorders, school problems (Fisher's exact test; $p<0.001)$ among motives, and parent loss experience $\left(\chi^{2}(1)=8.84 ; p<0.01\right)$ among socio-demographic characteristics were more often found in adolescents. Also, among socio-demographic characteristics, previous psychiatric history was more frequently noted in adults $\left(\chi^{2}(1)=4.41\right.$; $p<0.05)$.
Table 1 Demographic characteristics of all participants

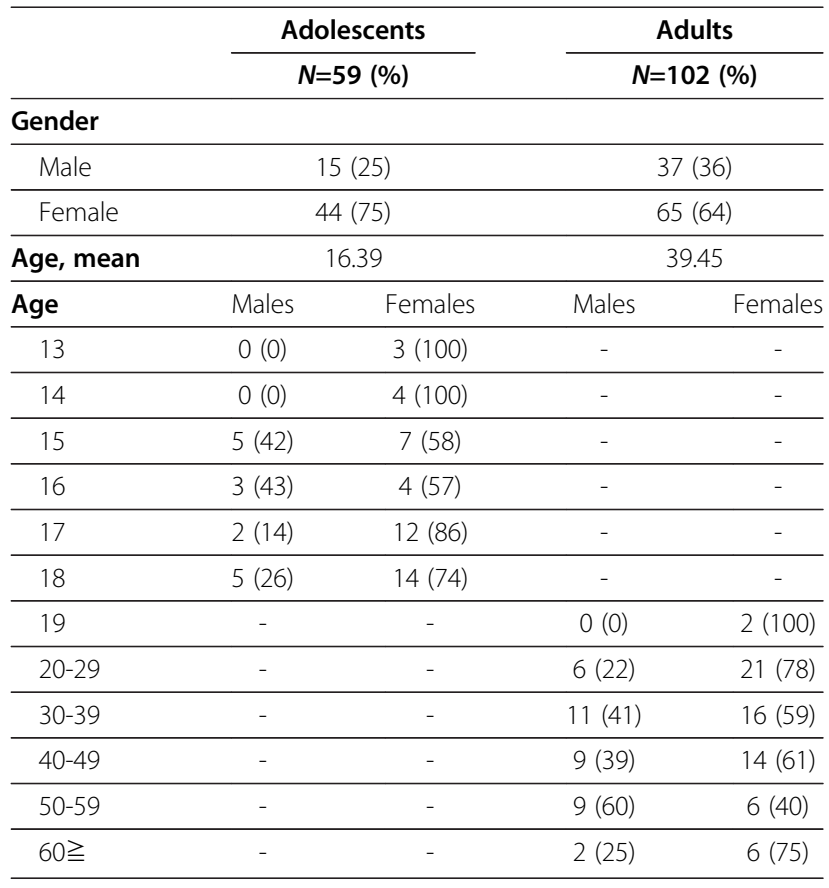

\section{Discussion}

This is the first study to examine the characteristics of serious suicide attempters among adolescents in Japan, focusing on psychiatric disorders, methods, motives and socio-demographic factors compared with adults.

We first examined the differences between 59 adolescent suicide attempters and 102 adults. The results revealed significant differences in BPD among psychiatric disorders, in financial problems and school problems among motives for suicide attempts, and in parent loss experience among socio-demographic characteristics.

As for the gender ratio among suicide attempters, the ratio of females was higher than that of males both in adolescents and adults. The subsequent gender-specific analysis revealed different characteristics between male adolescents and female adolescents, highlighting the fact that, if we compare adolescent suicide attempters with adult suicide attempters, we should also consider their characteristics according to gender.

\section{Psychiatric disorders}

A previous study reported that $95 \%$ of suicide attempters met the DSM-IV criteria for either axis I or axis II psychiatric diagnosis or both [30]. In the present study, psychiatric disorders were present in $98 \%$ of female adults, $93 \%$ of male adolescents, and $89 \%$ of both male adults and female adolescents. Therefore, among not only 
Table 2 Differences between adolescents and adults

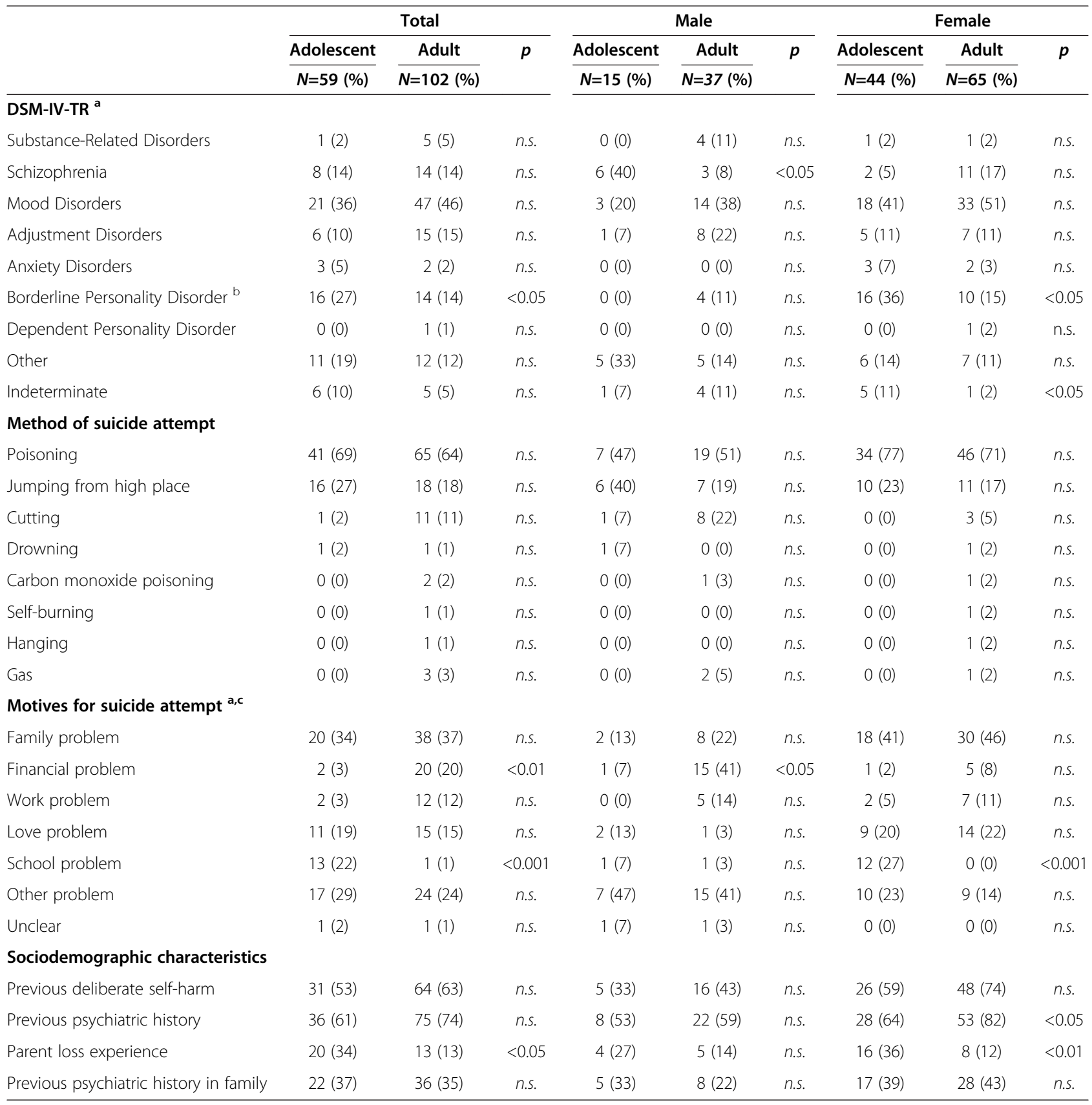

a There were repetitions in DSM-IV-TR and motives for suicide attempt. Therefore, some percentage totals are more than 100.

${ }^{b}$ Borderline Personality Disorders included borderline personality trait.

c We excluded psychiatric symptoms as motives for suicide attempt. Therefore, some percentage totals are less than 100.

adults but also adolescents, psychiatric disorders have a close relation with attempted suicide, and there may be psychiatric symptoms or social dysfunction with psychiatric disorders behind suicide.

Depressive disorders are consistently the most prevalent disorders among adolescent suicide victims [29]. In the present study, mood disorders were also most common among adolescents. However, according to the analysis by gender, schizophrenia was the most prevalent psychiatric disorder among male adolescents and was also seen more often in male adolescents than in male adults. The onset of schizophrenia is earlier in males than in females [31] and schizophrenia patients committing suicide are more often men and tend to be young [32]. According to a recent meta-analysis, $4.9 \%$ of patients with schizophrenia die of suicide, most of them soon after illness onset [33]. Additionally, those with schizophrenia tend to use highly lethal suicide methods 
[32]. The reason for male adolescents to have a high rate of schizophrenia in our study might be related to the gender difference at onset age of the condition. Additionally, as all participants in our study were in a medically serious and potentially fatal state, there may have been a selection bias. It is important that we identify male patients with schizophrenia as possible adolescent suicide attempters, because schizophrenia among adolescents increased the risk of suicide [34]. We also need to provide comprehensive initial treatment that improves adherence to early treatment via a psycho-education for patients and their families, as $2-11 \%$ of patients with schizophrenia attempt suicide at least once a year after starting treatment [35].

In females, BPD was more common among adolescents than adults. We especially focused on the diagnosis of personality disorders in adolescent participants, as adolescence is the developmental stage of personality. We investigated psychiatric symptoms in medical records from psychiatrist-conducted interviews of all participants or their parents as well as from information about any prior psychiatric treatments. Previous studies have reported that the frequency of BPD in female adolescents who committed suicide was $26 \%$ [36] and that it was $33 \%$ in individuals $15-29$ years old [37]. The result of the present study, that BPD was the second most common diagnosis for female adolescents, supported previous studies reporting that BPD was relatively frequently associated with suicide attempts and completed suicides among adolescents. Therefore, it is important to assess interpersonal relationships and emotional control of female adolescent suicide attempters with BPD. Likewise, it is necessary to examine possible developmental histories that might affect suicide attempts, focusing on the development of attachment, mother-child relationship problems, and traumatic episodes in childhood strongly associated with BPD. Indeterminate for psychiatric disorders also was more often seen among adolescents than adults. Some female adolescents may attempt suicide without any psychiatric symptoms and prior indications of emotional or behavioral problems. It has been pointed out that adolescents may be more impulsive than adults, and they may tend to focus on proximal consequences of behavior [38,39]. Therefore, we have to pay attention to impulses among female adolescents.

\section{Motives for suicide attempts and socio-demographic characteristics}

As for motives, family problems had the highest incidence in both male and female adolescents. The relation between family functioning and adolescent suicide attempts has been explored in many lines of research $[18,23]$. Our findings supported those previous studies. On the other hand, we found that adolescents, and particularly females, had more parent loss experience and school problems. This finding may indicate that female adolescents are more vulnerable to stresses in the immediate environment and interpersonal relationships than male adolescents and adults. Therefore, in regard to female adolescents, it is important to adequately assess family functioning and interpersonal conflict, and furthermore, to reach out to the patients and their families.

Male adults had significantly more financial problems than male adolescents, while there was no difference between females. A previous study in Japan [40] reported that unemployment is an important suicidal risk factor in males. They may suffer more severe effects from failures on the job or struggles to survive from such as debts. For male adult suicide attempters with financial problems, they should be paid close attention, and adequate intervention with a view to solving their financial problems should be undertaken.

In terms of previous psychiatric history, female adults had significantly more than female adolescents, while there was no difference in males. This may indicate that the frequency of psychiatric care is greater in females than males and that the tendency becomes stronger with increasing age. Still, despite receiving psychiatric care in the past, more than half of all participants attempted suicide. If suicide attempters discontinue their psychiatric treatment, it is vital that their treatment be re-established. Even if they are undergoing psychiatric treatment, they may have poor medication adherence, severe psychiatric symptoms, and psychosocial impairments. Therefore, for the prevention of subsequent suicide attempts, we have to determine their psychosocial issues and remedy psychosocial dysfunctions in parallel with improving adherence and psychiatric symptoms as part of their psychiatric treatment.

\section{Limitations}

This study has several limitations. First, it used a relatively small sample size, meaning that our results can only be generalized with caution. However, this study sample does present meaningful data, in consideration of measures to combat suicide, because all participants were serious suicide attempters with characteristics similar to complete suicide attempters. Another limitation is that the study period was different between adolescents and adults, and we could not control the differences between the time periods. A third limitation is that the psychiatric diagnoses of all participants were reached without structured interviews and assessment tools. It is difficult for psychiatrists to conduct structured and systematic interviews for suicide attempters because of the shorter hospitalization and temporal constraints in 
CCMC. We did our best to enhance the reliability of the psychiatric diagnoses, holding discussions in the case of disagreement with the psychiatric diagnoses. However, there may be an issue that the diagnoses in the present work are more studied, as they were based more on mental status records than diagnoses that are obtained by direct and structured questionnaires.

We did not divide adolescents or adults into subgroups according to age, such as younger adolescents or elderly adults, as our small sample size made it difficult to compare the different age generations in detail. Thus, in the present study, we just aimed to explore the robust differences between adolescents and adults. However, in a future study based on a larger study population we will have to investigate the differences among the various age groups from adolescents to adults. Moreover, it will be necessary to conduct a prospective longitudinal study by means of structured assessment tools and develop interventions tailored to adolescent characteristics.

\section{Conclusion}

Our present findings indicate that adolescent attempters were more frequently diagnosed with BPD and had more school problems and parent loss experience but had less financial problems. Additionally, by gender, we found the importance of identifying patients with schizophrenia especially among male adolescents, because they are more commonly diagnosed with this disorder. Furthermore, this study has revealed that female adolescents more often receive a diagnosis of BPD, and they have more parent loss experience and school problems, indicating the importance of adequately assessing family functioning and interpersonal conflicts for female adolescents.

\section{Abbreviations}

CCMC: Critical Care Medical Center; DSM: Diagnostic and Statistical Manual of Mental Disorders; BPD: Borderline Personality Disorder.

\section{Competing interests}

The authors declare that they have no competing interests.

\section{Authors' contributions}

All authors contributed to study design. YK, TI, RN contributed to data collection. YK, RN, TS, YO wrote the analysis plan. YK conducted the statistical analysis and wrote the first draft of the manuscript. TI, RN, TS, YO discussed the ideas in the manuscript and contributed to its preparation. All authors read and approved the final manuscript.

\section{Acknowledgements}

This study was supported by Health Labor Sciences Research Grants from the Ministry of Health Labor and Welfare. We would like to thank the staff of the Department of Critical Care Medicine of Nippon Medical School.

Received: 21 April 2012 Accepted: 2 October 2012

Published: 8 November 2012

\section{References}

1. National Police Agency: Statistics of suicide victims in Japan in 2004 National Police Agency Japan 2005, in Japanese.
2. World Health Organization: Country reports and charts available. http://www.who.int/mental_health/prevention/suicide/country_reports/en/ index.html.

3. National Police Agency: Statistics of suicide victims in Japan in 2010 National Police Agency Japan 2011, in Japanese).

4. Ministry of Health, Labour and Welfare: Vital statistics of Japan 2010. http://www.mhlw.go.jp/toukei/saikin/hw/jinkou/geppo/nengai10/dl/ h22_gaikyou.pdf.

5. Desapriya EB, Iwase N: New trends in suicide in Japan. Inj Prev 2003, 9(3):284.

6. Bridge JA, Goldstein TR, Brent DA: Adolescent suicide and suicidal behavior. J Child Psychol Psychiatry 2006, 47(3-4):372-394.

7. Hidaka Y, Operario D, Takenaka M, et al: Attempt suicide and associated risk factors among youth in urban Japan. Soc Psychiatry Psychiatr Epidemiol 2008, 43(9):752-757.

8. Katsumata $Y$, Matsumoto T, Kitani M, et al: Electronic media use and suicidal ideation in Japanese adolescents. Psychiatry Clin Neurosci 2008 62(6):744-746

9. Matsumoto T, Imamura F, Chiba Y, et al: Prevalences of lifetime histories of self-cutting and suicidal ideation in Japanese adolescents: differences by age. Psychiatry Clin Neurosci 2008, 62(3):362-364.

10. Haukka J, Suominen K, Partonen T: Determinants and outcomes of serious attempted suicide: a nationwide study in Finland, 1996-2003. Am J Epidemiol 2008, 167(10):1155-1163

11. Hischfeld RM, Russell JM: Assessment and treatment of suicidal patients. N Engl J Med 1997, 337(13):910-915

12. Tidemalm D, Langstrom $N$, Lichtenstein P: Risk of suicide after suicide attempt according to coexisting psychiatric disorder: Swedish cohort study with long term follow-up. BMJ 2008, 337:a2205.

13. Dube SR, Anda RF, Felitti VJ, et al: Childhood abuse, household dysfunction, and the risk of attempted suicide throughout the life span: findings from the Adverse Childhood Experiences Study. JAMA 2001, 286(24):3089-3096.

14. Qin P, Agerbo E, Mortensen PB: Suicide risk in relation to family history of completed suicide and psychiatric disorders: a nested case-control study based on longitudinal registers. Lancet 2002, 360(9340):1126-1130.

15. Beautrais $A$ : Subsequent mortality in medically serious suicide attempts: a 5 year follow-up. Aust N Z J Psychiatry 2003, 37(5):595-599.

16. Nordstrom P, Samuelson M, Asberg M: Survival analysis of suicide risk after attempted suicide. Acta Psychiatr Scand 1995, 91(5):336-340.

17. Owens D, Horrocks J, House A: Fatal and non fatal repetition of self-harm. Systematic review. Br J Psychiatry 2002, 181:193-199.

18. Spirito A, Brown L, Overholser J, et al: Attempted suicide in adolescence: $A$ review and critique of the literature. Clin Psychol Rev 1989, 9(3):335-363.

19. Shaffer D, Piacentini J: Suicide and attempted suicide. In Child psychiatry: Modern approaches. 3rd edition. Edited by Rutter M, Taylor E. Oxford: Blackwell Scientific; 1994:407-424.

20. Beautrais A: Suicide and serious suicide attempts in youth: a multiple-group comparison study. Am J Psychiatry 2003, 160(6):1093-1099.

21. Lewis M: Dulcan MK. In Textbook of Child and Adolescent Psychiatry. 3rd edition. Edited by Wiener JM. America: American Psychiatric Publishing; 2003:13-44.

22. Kerr D, Preuss L, King C: Suicidal adolescents' social support from family and peer: gender-specific associations with psychopathology. J Abnorm Child Psychol 2006, 34(1):103-114.

23. Daniel S, Goldston D: Interventions for suicidal youth: a review of the literature and developmental considerations. Suicide Life Threat Behav 2009, 39(3):252-268.

24. Weisz J, Hawley K: Developmental factors in the treatment of adolescents. J Consult Clin Psychol 2002, 70(1):21-43.

25. Kurosawa $H$, Iwasaki $Y$, Watanabe $N$, et al: The practice of consultationliaison psychiatry in Japan. Gen Hosp Psychiatry 1993, 15(3):160-165.

26. Kishi $Y$, Iwasaki $Y$, Takezawa $K$, et al: Delirium in critical care unit patients admitted through an emergency room. Gen Hosp Psychiatry 1995, 17(5):371-379.

27. Nock MK, Borges G, Bromet EJ, et al: Suicide and suicidal behavior. Epidemiol Rev 2008, 30:133-154

28. Posner K, Oquendo MA, Gould M: Columbia Classification Algorithm of Suicide Assessment (C-CASA): classification of suicidal events in the FDA's pediatric suicidal risk analysis of antidepressants. Am J Psychiatry 2007, 164(7):1035-1043. 
29. Gould MS, Greenberg T, Velting DM, et al: Youth suicide risk and preventive interventions: a review of the past 10 years. J Am Acad Child Adolesc Psychiatry 2003, 42(4):386-405.

30. Yamada T, Kawanishi C, Hasegawa $\mathrm{H}$, et al: Psychiatric assessment of suicide attempters in Japan: a pilot study at a critical emergency unit in an urban area. BMC Psychiatry 2007, 7:64-70.

31. Takahashi S, Matsuura M, Tanabe E, et al: Age at onset of schizophrenia: gender differences and influence of temporal socioeconomic change. Psychiatry Clin Neurosci 2000, 54(2):153-156.

32. Breier A, Astrachan BM: Characterization of schizophrenic patients who commit suicide. Am J Psychiatry 1984, 141(2):206-209.

33. Palmer BA, Pankratz VS, Bostwick JK: The lifetime risk of suicide in schizophrenia: a reexamination. Arch Gen Psychiatry 2005, 62(3):247-253.

34. Joa I, Johannessen JO, Langeveld J, et al: Baseline profiles of adolescent vs. adult-onset first-episode psychosis in an early detection program. Acta Psychiatr Scand 2009, 119(6):494-500.

35. Melle L, Johannessen JO, Friis $\mathrm{S}$, et al: Course and predictors of suicidality over the first two years of treatment in first-episode schizophrenia spectrum psychosis. Arch Suicide Res 2010, 14(2):158-170.

36. Marttunen MJ, Henriksson MM, Aro HM, et al: Suicide among female adolescents: characteristics and comparison with males in the age group 13 to 22 years. J Am Acad Child Adolesc Psychiatry 1995, 34(10):1297-1307.

37. Runeson B, Beskow J: Borderline personality disorder in young Swedish suicides. J Nerv Ment Dis 1991, 179(3):153-156

38. Nurmi JE: How do adolescents see their future? A review of the development of future orientation and planning. Dev Rev 1991, 11(1):1-59.

39. Reyna V, Farley F: Risk and rationality in adolescent decision making: implications for theory, practice, and public policy. Psychological Science in the Public Interest 2006, 7(1):1-44.

40. Inoue $\mathrm{K}$, Tanii $\mathrm{H}$, Abe $\mathrm{S}$, et al: The report in the correlation between the factor of unemployment and suicide in Japan. Am J Forensic Med Pathol 2008, 29(2):202-203.

doi:10.1186/1471-244X-12-191

Cite this article as: Kawashima et al:: The characteristics of serious suicide attempters in Japanese adolescents- comparison study between adolescents and adults. BMC Psychiatry 2012 12:191.

\section{Submit your next manuscript to BioMed Central and take full advantage of:}

- Convenient online submission

- Thorough peer review

- No space constraints or color figure charges

- Immediate publication on acceptance

- Inclusion in PubMed, CAS, Scopus and Google Scholar

- Research which is freely available for redistribution 